PAPER

\title{
Brain activation during ideomotor praxis: imitation and movements executed by verbal command
}

\author{
M Makuuchi, T Kaminaga, M Sugishita
}

J Neurol Neurosurg Psychiatry 2005;76:25-33. doi: 10.1136/jnnp.2003.029165

See end of article for authors' affiliations

Correspondence to: Professor M Sugishita, Institute of Brain and Blood Vessels, 366 Ohtamachi, Isesaki, Gunma 372-0006, Japan; morihiro_sugi@ ybb.ne.jp

Received 27 September 2003 In revised form 14 April 2004 Accepted 18 April 2004
Background: Ideomotor apraxia is a disorder of both imitation movements and movements executed by verbal command. Lesion studies have identified the left parietal lobe as the neural correlate for ideomotor praxis (IP), although there are opposing views.

Objectives: To localise the neural substrates for IP using functional magnetic resonance imaging. Brain regions activated by both imitation and verbal command movements were tested against a simple self paced movement.

Methods: Twenty two young, right handed, healthy subjects were examined. Functional and anatomical data were acquired. The experiment comprised three motor conditions (imitation, movements executed by verbal command, and finger bending/unbending) and a rest condition. All motor tasks were performed using the left hand. Eighteen drawings of left hand postures were presented for the imitation condition. Identical postures were instructed verbally for the verbal command condition. The finger bending/ unbending movement was self paced. Blood oxygenation level dependent (BOLD) signal increases were compared during two kinds of IP (imitation and verbal command movements) and during finger bending/ unbending movements.

Results: The depth of the posterior part of the left intraparietal sulcus and bilateral precunei were activated during both imitation and verbal command movements. The difference in BOLD signal between imitation and verbal command movements was localised in the dorsal and ventral occipital areas. BOLD signal differences for movements executed by verbal command against imitation were observed in the superior temporal areas.

Conclusions: The depth of the posterior part of the left intraparietal sulcus and bilateral precunei are the neural substrates for IP.
$\mathrm{T}$ he concept of ideomotor apraxia was proposed by Liepmann. ${ }^{1}$ Operationally, ideomotor apraxia is defined as "failure to produce the correct movement in response to verbal command, or failure to imitate correctly a movement performed by the examiner" that cannot be explained by "weakness, incoordination, akinesia, abnormal reflexes, impaired auditory comprehension, or impaired visual perception". ${ }^{2}$ For example, individuals with ideomotor apraxia cannot make correct motor responses when the examiner asks them to salute or to wave their hands to express "goodbye", or when requested to imitate the movements of the examiner. Because most patients with ideomotor apraxia have a deficit both in imitation and in movements executed by verbal command, the brain region activated by both imitation and movements executed by verbal command is thought to contain the neural substrate for ideomotor praxis.

The neural correlates of ideomotor apraxia have been thought to be located in the left parietal lobe, but some studies have claimed other regions. Lesion studies have reported defective imitation and movements executed by verbal commands (ideomotor praxis) after left parietal damage. ${ }^{3-7}$ A recent lesion study found maximum overlap of lesions in patients with ideomotor apraxia in the left intraparietal sulcus. ${ }^{8}$ However, a study of a large sample claimed a crucial role for deep parietofrontal and occipitofrontal fibres in producing ideomotor apraxia, challenging the traditional view. ${ }^{9}$ A meta-analysis found that lesions to deep subcortical structures, such as the lenticular nucleus or putamen, with involvement of periventricular or peristriatal white matter, can cause ideomotor apraxia. ${ }^{10}$ Neuroimaging studies have reported that the neural substrate for imitation lies in the left Brodmann area 44, alias Broca's area. ${ }^{112}$
Movements executed by verbal command require the subjects to recall what to do, a cognitive process that is not contained in imitation. In contrast, imitation requires the subject to recognise and remember what he/she sees, which is not required in movements executed by verbal command. Indeed, the performances of patients with ideomotor apraxia in response to verbal command or imitation can be different; in some cases, performance by imitation is better than by verbal command..$^{13}$ In contrast, in some other cases, performance by verbal command is easier than imitation. ${ }^{6}$ No evidence is available concerning the different brain mechanisms involved in these two ideomotor praxis.

This functional magnetic resonance imaging (fMRI) study was designed to reveal the brain regions that commonly subserve two ideomotor praxis-imitation and movements executed by verbal command-and to find the difference in activated regions between the two types of ideomotor praxis. The subject was requested to make hand postures using the left hand by imitation and by verbal command. A finger bending/unbending movement condition was introduced as a base line condition, against which we detected blood oxygenation level dependent (BOLD) signal increases in the two ideomotor praxis conditions.

\footnotetext{
Abbreviations: BOLD, blood oxygenation dependent; EPI, echo planar imaging; $f M R I$, functional magnetic resonance imaging; $F B / U M$, finger bending/unbending movement; FOV, field of view; I, imitation; MNI, Montreal Neurological Institute; MRI, magnetic resonance imaging; PET, positron emission tomography; SPM, statistical parametric mapping; VC, verbal command
} 


\section{METHODS}

\section{Subjects}

Twenty two (20 men and two women) young, right handed, healthy subjects were examined (mean age, 22.9 years; SD, 3.70; range, 19-37). Handedness was assessed by the Edinburgh inventory (mean, 97; SD, 4.8; range, 84-100). ${ }^{14}$ The experimental procedures were approved by the research ethics committee of the faculty of medicine, University of Tokyo, and written informed consent was received from all the subjects.

\section{Stimuli}

All 18 hand postures, adapted from Sperry, ${ }^{15}$ were of the left hand, and were drawn in black lines on a white background. The verbal commands were names of things represented by the hand postures or directions of the finger/wrist configurations. The verbal commands were as follows; stone, scissors, paper, OK, rake, bud, gun, fox, four fingers, three fingers, bend outside, bend inside, extend the forefinger, extend the little finger, extend the thumb, hide the thumb, arch the middle finger to the thumb, and arch the ring finger to the thumb (fig 1). The pictures of the hand postures were back projected on to a screen from an LCD projector (Panasonic TH-L592, Japan) and the subject viewed them though a half mirror attached to the head coil. Verbal commands and auditory cues were delivered to both ears through air tube earphones. Presentation of the stimuli was controlled by Psyscope 1.1 software (Department of Psychology, Carnegie Mellon University, Pittsburgh, PA, USA, 1994) on a Macintosh computer.

\section{Tasks}

The experiment comprised three motor conditions (imitation (I), verbal command (VC), finger bending/unbending movement $(\mathrm{FB} / \mathrm{UM}))$ and one rest condition (REST). The subject was required to perform all of the motor tasks using the left hand, because clinical tests for ideomotor apraxia in the literature have frequently been carried out only in the left hand because the right hand is often paralysed in patients with apraxia. Clinical tests of ideomotor apraxia include motor tasks using various parts of the body, but only the hand was used in this fMRI study because of the limited space in the magnetic resonance imaging (MRI) bore and to avoid possible head movement of the subject. Given such restricted settings, the use of the hand is the best choice, because it affords a broad variety of shapes and avoids habituation effects in the BOLD signal. Two postures used in our study, stone and paper, appeared in Liepmann's apraxia test. $^{16}$ The subject lay supine on the gantry of the MRI scanner with the left forearm upright and supported at the elbow, the left hand positioned above the trunk, and the right arm laid straight along the trunk. During all of the conditions, the subject was able to see his left hand if he needed.

\section{Imitation (I)}

The first task was to imitate the hand postures using the left hand (fig 1). Nine line drawings representing hand postures were presented on the screen with the projector one by one every four seconds, and the subject mimicked the postures with the left hand. The duration of the condition was 36 seconds. No auditory stimuli were presented.

\section{Verbal command (V)}

The second task was to make hand postures according to verbal commands. Through the earphones, nine commands were delivered one by one in a male voice every four seconds. The subject was required to form the indicated hand posture and maintain it until hearing the next command. The set of hand postures used in the verbal command condition was identical to the set of postures used in the imitation condition. A blank white screen was presented during this condition, which lasted 36 seconds.

\section{Finger bending/unbending movement ( F)}

The third task was to bend all the fingers sequentially from the thumb to the little finger and to unbend them in reverse order. The finger bending/unbending movement was performed repeatedly at the subject's own pace until the end of the condition. We instructed the subject how to bend/unbend the fingers by showing the movement (one cycle took about two seconds), which prevented an extremely fast or slow pace. The start and end of the condition were announced by auditory cues of "finger movement" and "stop" in a male
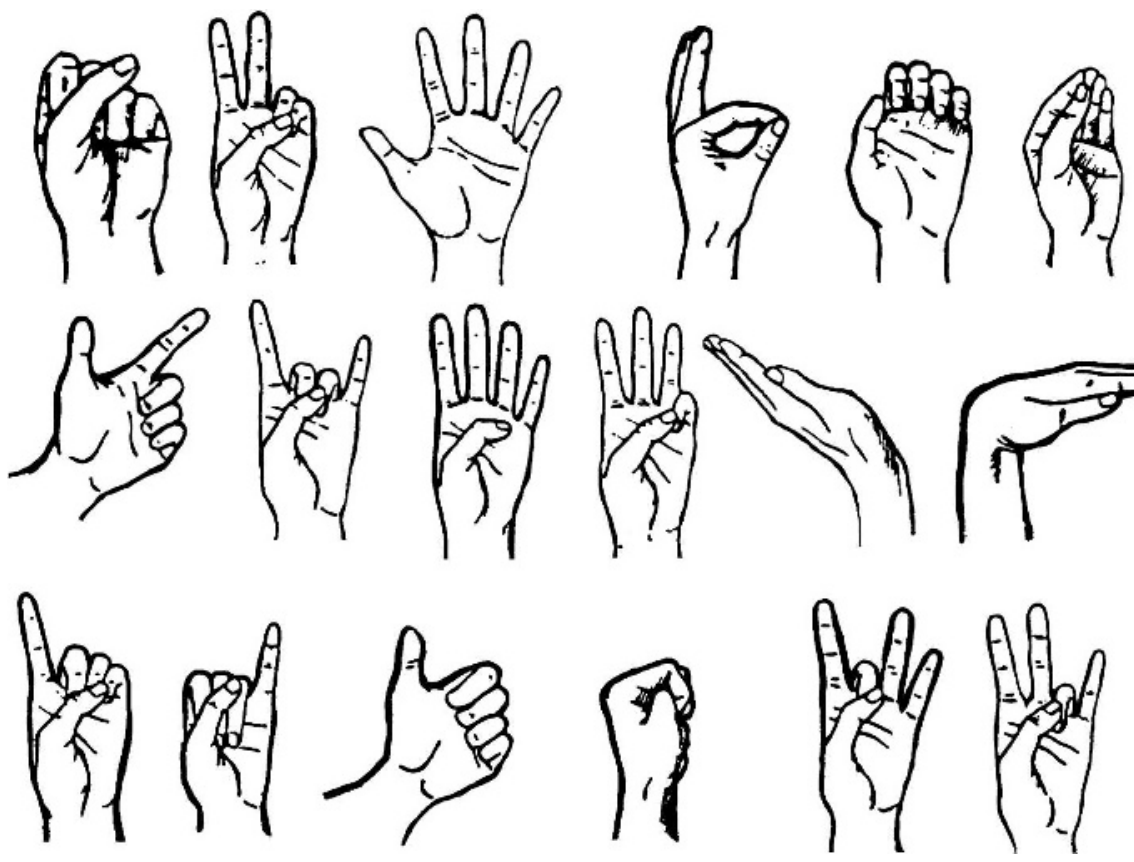

Figure 1 The hand posture visual stimuli were taken from Sperry. ${ }^{15}$

Verbal commands were given as names of objects or directions of the finger/ wrist configurations. From the top left: stone, scissors, paper, OK, rake, bud, gun, fox, four fingers, three fingers, bend outside, bend inside, extend the forefinger, extend the little finger, extend the thumb, hide the thumb, arch the middle finger to the thumb, arch the ring finger to the thumb. 

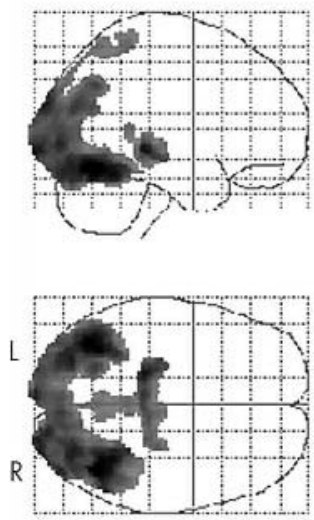

C
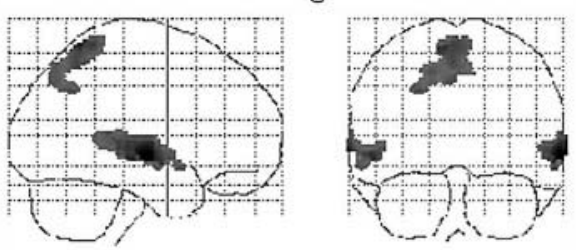
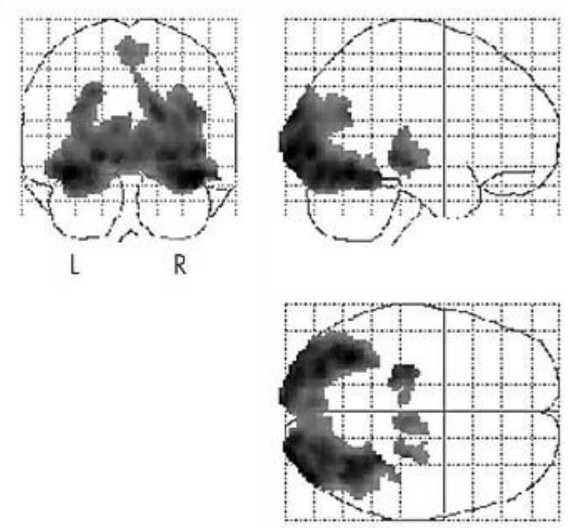

Figure 2 Statistical parametric maps of $z(S P M\{Z\} s)$ of the neural substrates of imitation and of movements executed by verbal command. SPM $\{Z\}_{s}$ are thresholded at $p<0.05$ (corrected for multiple comparisons). (A) Activation revealed by the detection of signal increase in I against FB/UM, masked by I against REST. (B) Activation revealed by the detection of signal increase in I against VC, masked by I against REST. (C) Activation revealed by the detection of signal increase in VC against $F B / U M$, masked by $V C$ against REST. (D) Activation revealed by the detection of signal increase in VC against I, masked by VC against REST. The SPM $\{Z\}$ s are displayed on a glass brain viewed from three directions. The transverse projection was viewed from the top, and the coronal projection was viewed from the back (the right of the map corresponds to the right of the brain). Tables 1-4 summarise the local maxima of the SPM $\{Z\} \mathrm{s}$. FB/UM, finger bending/unbending movement; I, imitation; VC, verbal command.
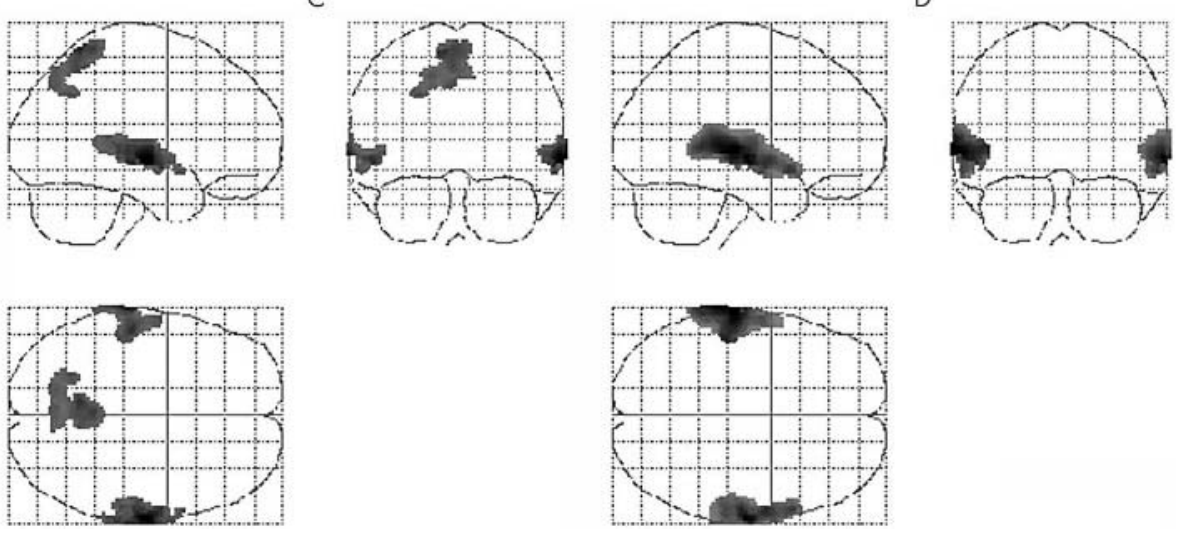

voice through the earphones. Except for the cues to start and to stop, there were no auditory stimuli. A blank white screen was presented throughout the condition. The duration of the condition was 36 seconds.

\section{Rest $(\operatorname{Re})$}

Rest conditions were inserted between movement conditions. In this condition, the subject was asked to look at the blank screen and to relax. The duration of the condition was 36 seconds, during which a blank white screen was presented. No auditory stimuli were provided.

The three movement conditions were repeated three times in a pseudo-random order with intervening rest conditions. The order of condition presentation was counterbalanced among subjects. Before the scan, the subject saw all of the pictures used in the experiment. Then they had a short practice with the visual and auditory stimuli to become accustomed to the tasks. We observed very good performance $(>95 \%)$ of the tasks in the practice sessions and all subjects reported their almost perfect performances in the scanning sessions.

\section{Data acquisition}

Echo planar imaging (EPI) data were acquired with a $1.5 \mathrm{~T}$ Signa Horizon MRI scanner (GE, Waukesha, Wisconsin, USA). The whole brain was covered with 18 axial images without slice gaps (time of repetition $(\mathrm{TR})=4.0 \mathrm{sec}$, time of echo $(\mathrm{TE})=50 \mathrm{msec}$, field of vision $(\mathrm{FOV})=24 \times 24 \mathrm{~cm}^{2}$, $64 \times 64$ pixels, $8 \mathrm{~mm}$ thick). A total of 176 volumes were acquired over 11 minutes and 44 seconds, including four dummy volumes at the beginning to allow time for magnetic saturation. Structural high resolution $\mathrm{Tl}$ images were also

collected before or after the experimental sessions $\left(\mathrm{FOV}=24 \times 24 \mathrm{~cm}^{2}, \quad 256 \times 256\right.$ matrix, 124 slices, $1.3 \mathrm{~mm}$ thick). The forehead and the chin were fixed with straps, and an air cushion was placed around the head to prevent head motion.

\section{Analysis}

Data analysis was carried out using SPM96 software ${ }^{17-20}$ implemented in Matlab (Mathworks Inc, Natick, Massachusetts, USA) on a SUN Ultra 2 workstation. As a preprocessing step, the EPI images were realigned to the first one. A mean EPI image was created for corregisteration of the EPI images to the individual $\mathrm{Tl}$ image. Individual $\mathrm{Tl}$ images were normalised to the Montreal Neurological Institute (MNI) template. ${ }^{21}$ This process transformed the $\mathrm{Tl}$ and all the EPI images into the MNI stereotaxic space, ${ }^{21}$ which is similar to, but slightly different from, the Talairach space, ${ }^{22}$ to allow comparisons among studies or subjects, or multisubject analysis. The EPI images were resampled into $2 \times 2 \times 2 \mathrm{~mm}^{3}$ voxels. To improve the signal to noise ratio and to compensate for the anatomical variability of individual brains, all volumes were smoothed with a full width at half maximum, $8 \mathrm{~mm}$ isotropic Gaussian kernel before the statistical calculation.

Statistical analysis was performed according to the mixed effect model, ${ }^{23}$ in which the intersubject component of variance and the intrasubject component of variance are taken into account. In practice, a two level process was used. First, activation for each task was estimated in each subject separately. The haemodynamic response was modelled with a boxcar function convolved with a haemodynamic response function, and mean signal intensity during each task was estimated according to the general linear model. Shifts 


\begin{tabular}{|c|c|c|c|c|c|}
\hline \multirow[b]{2}{*}{ Activated region } & \multirow[b]{2}{*}{$L / R$} & \multicolumn{3}{|c|}{ Coordinates } & \multirow[b]{2}{*}{$Z$ value } \\
\hline & & $x$ & $y$ & $z$ & \\
\hline Inferior occipital gyrus & $\mathrm{R}$ & 26 & -96 & -4 & 5.96 \\
\hline \multirow{2}{*}{ Middle occipital gyrus } & $\mathrm{L}$ & -30 & -90 & 10 & 4.60 \\
\hline & $\mathrm{R}$ & 42 & -84 & 0 & 5.84 \\
\hline \multirow{2}{*}{ Fusiform gyrus } & $\mathrm{L}$ & -38 & -66 & -14 & 6.82 \\
\hline & $\mathrm{R}$ & 42 & -60 & -16 & 6.79 \\
\hline \multirow{2}{*}{ Lingual gyrus } & $\mathrm{L}$ & -4 & -84 & -2 & 4.63 \\
\hline & $\mathrm{R}$ & 6 & -82 & -2 & 5.11 \\
\hline \multirow{2}{*}{ Cuneus } & L & -6 & -100 & -4 & 5.59 \\
\hline & $\mathrm{R}$ & 4 & -76 & 44 & 3.58 \\
\hline Intraparietal sulcus & L & -30 & -70 & 28 & 4.77 \\
\hline \multirow[t]{3}{*}{ Precuneus } & L & -6 & -56 & 66 & 3.55 \\
\hline & $\mathrm{R}$ & 8 & -58 & 62 & 3.55 \\
\hline & & 2 & -70 & 52 & 3.31 \\
\hline \multirow[t]{4}{*}{ LGN } & L & -20 & -30 & -4 & 6.07 \\
\hline & & -22 & -30 & -4 & 5.46 \\
\hline & & -26 & -22 & -8 & 5.42 \\
\hline & $\mathrm{R}$ & 26 & -22 & -8 & 4.18 \\
\hline \multirow[t]{2}{*}{ Superior colliculus } & L & -6 & -30 & -4 & 5.20 \\
\hline & $\mathrm{R}$ & 8 & -28 & -4 & 4.65 \\
\hline Pulvinar & $\mathrm{R}$ & 22 & -28 & 0 & 4.28 \\
\hline Red nucleus & $\mathrm{R}$ & 6 & -20 & -2 & 3.94 \\
\hline \multirow[t]{2}{*}{ Midbrain } & L & -8 & -24 & -10 & 3.61 \\
\hline & $\mathrm{R}$ & 8 & -26 & -8 & 4.45 \\
\hline
\end{tabular}

caused by global signal changes were removed by applying a high pass filter (cutoff cycle, $144 \mathrm{sec}$ ) and proportional scaling. Then, estimates were compared in groups by the paired $t$ test, taking into account the variance of activation estimated among the subjects. Creating four mean volumes for each condition for each subject, we analysed a total of 88 volumes in the mixed effect analysis. After transformation of the $\mathrm{t}$ value to a $\mathrm{Z}$ value, a statistical parametric map of $\mathrm{Z}$ $(\operatorname{SPM}\{Z\})$ was created. Evaluation of activation was thresholded at uncorrected $\mathrm{p}<0.001$ for the $\mathrm{Z}$ value of each voxel, then corrected for multiple non-independent comparisons by the Gaussian random field theory ${ }^{20}$ at $\mathrm{p}<0.05$.

A statistical analysis identifying regions with significant signal increases in condition A compared with condition B is referred to as "detection of signal increase in A against B". We performed six analyses, which are described below in two sections. An inclusive mask analysis was applied, to avoid finding false activation caused by deactivation (signal intensity decrease relative to the REST condition) in contrasted conditions. The threshold for the pass criterion of the mask was set to $\mathrm{p}<0.05$ (not corrected).

First, we identified the regions that had significant signal increases in imitation and in movements executed by verbal command (analysis 1-4). The neural substrates of imitation were identified by analysis 1 and 2. Detection of signal increase in I against FB/UM, masked by I against REST showed brain regions that were activated by imitation, but eliminated the regions that were also activated by the finger bending/unbending movement (analysis 1). By confining the activated region of signal increase in I against $\mathrm{FB} / \mathrm{UM}$ within the activated regions of signal increase in I against REST, we exclude the possibility of counting false activation caused by negative activity in the FB/UM condition. The detection of signal increase in I against VC, masked by I against REST showed brain regions that were activated by imitation, but eliminated regions that were also activated by the movements executed by verbal command (analysis 2).

Table 2 The local maxima of activated regions in I against VC, masked by I against REST

\begin{tabular}{|c|c|c|c|c|c|}
\hline \multirow[b]{2}{*}{ Activated region } & \multirow[b]{2}{*}{$L / R$} & \multicolumn{3}{|c|}{ Coordinates } & \multirow[b]{2}{*}{$Z$ value } \\
\hline & & $\mathbf{x}$ & $y$ & $\mathbf{z}$ & \\
\hline \multirow[t]{2}{*}{ Fusiform gyrus } & $\mathrm{L}$ & -34 & -58 & -14 & 6.71 \\
\hline & $\mathrm{R}$ & 34 & -58 & -14 & 6.27 \\
\hline Lingual gyrus & $\mathrm{L}$ & -6 & -82 & -8 & 4.70 \\
\hline \multirow{2}{*}{ Inferior occipital gyrus } & $\mathrm{L}$ & -24 & -62 & 38 & 6.90 \\
\hline & $\mathrm{R}$ & 30 & -96 & -2 & 6.90 \\
\hline \multirow[t]{2}{*}{ Middle occipital gyrus } & $\mathrm{L}$ & -38 & -68 & -16 & 6.35 \\
\hline & $\mathrm{R}$ & 42 & -84 & 0 & 6.71 \\
\hline Middle temporal gyrus & $\mathrm{R}$ & 40 & -62 & 22 & 4.60 \\
\hline Hippocampal gyrus & $\mathrm{R}$ & 36 & -32 & -18 & 3.96 \\
\hline \multirow[t]{3}{*}{ LGN } & L & -22 & -30 & -4 & 5.46 \\
\hline & & -26 & -22 & -8 & 5.42 \\
\hline & $\mathrm{R}$ & 28 & -24 & -4 & 4.80 \\
\hline Pulvinar & $\mathrm{R}$ & 24 & -14 & -2 & 3.30 \\
\hline Medial pallidum & $\mathrm{R}$ & 22 & -14 & -2 & 3.30 \\
\hline \multirow{2}{*}{ Midbrain } & $\mathrm{L}$ & -8 & -24 & -10 & 3.61 \\
\hline & $\mathrm{R}$ & 8 & -26 & -8 & 4.45 \\
\hline
\end{tabular}


Table 3 The local maxima of activated regions revealed in VC against FB/UM, masked by VC against REST

\begin{tabular}{|c|c|c|c|c|c|}
\hline \multirow[b]{2}{*}{ Activated region } & \multirow[b]{2}{*}{$L / R$} & \multicolumn{3}{|c|}{ Coordinates } & \multirow[b]{2}{*}{$Z$ value } \\
\hline & & $\mathbf{x}$ & $y$ & $\mathbf{z}$ & \\
\hline Superior temporal gyrus & $\mathrm{L}$ & $\begin{array}{l}-68 \\
-54 \\
-60\end{array}$ & $\begin{array}{l}-28 \\
-22 \\
-8\end{array}$ & $\begin{array}{l}0 \\
-4 \\
-8\end{array}$ & $\begin{array}{l}4.78 \\
4.47 \\
3.57\end{array}$ \\
\hline Superior temporal gyrus & $\mathrm{R}$ & $\begin{array}{l}66 \\
68 \\
58\end{array}$ & $\begin{array}{l}-14 \\
-26 \\
-30\end{array}$ & $\begin{array}{l}-2 \\
6 \\
0\end{array}$ & $\begin{array}{l}5.70 \\
4.28 \\
4.01\end{array}$ \\
\hline Precuneus & L & $\begin{array}{l}-8 \\
-2 \\
-2 \\
-4 \\
2\end{array}$ & $\begin{array}{l}-54 \\
-46 \\
-58 \\
-70 \\
-70\end{array}$ & $\begin{array}{l}66 \\
66 \\
54 \\
44 \\
52\end{array}$ & $\begin{array}{l}4.52 \\
4.21 \\
3.96 \\
3.75 \\
3.63\end{array}$ \\
\hline Intraparietal sulcus & $\mathrm{L}$ & $\begin{array}{l}-24 \\
-16\end{array}$ & $\begin{array}{l}-60 \\
-70\end{array}$ & $\begin{array}{l}38 \\
50\end{array}$ & $\begin{array}{l}3.76 \\
3.66\end{array}$ \\
\hline
\end{tabular}

$\mathrm{FB} / \mathrm{UM}$, finger bending/unbending movement; L, left; R, right; VC, verbal command.

Brain regions engaged in movements executed by verbal command were revealed by analyses 3 and 4 . Detection of signal increase in VC against FB/UM, masked by VC against REST showed regions that were activated by movements executed by verbal command, eliminating regions that were also activated by the finger bending/unbending movement (analysis 3). Detection of signal increase in VC against I, masked by VC against REST showed regions that were activated by movements executed by verbal command, and eliminating regions that were also activated by imitation (analysis 4).

Second, we detected signal increases in the mean of two ideomotor praxis conditions against the FB/UM condition, and detected signal increase in reverse comparison by analyses 5 and 6 . Our main interest was to find the neural substrates of ideomotor praxis, which were activated in common by imitation and by movements executed by verbal command. This was identified by signal increases in (I + VC)/ 2 against FB/UM, masked by I against REST, VC against REST, I against FB/UM, and VC against FB/UM (analysis 5). The regions that were activated in only one of the two ideomotor praxis conditions were excluded by the masks that passed only commonality of activated regions for all four analyses. This method has been applied in other studies. ${ }^{24} 25$ The regions activated for the finger bending/unbending movement were identified by signal increases in FB/UM against $(\mathrm{I}+\mathrm{VC}) / 2$, masked by FB/UM against REST, FB/UM against I, and FB/UM against VC (analysis 6). This analysis was the reverse of analysis 5 . Masks that passed only the conjunction of activated regions for all three analyses were applied.

The FB/UM condition was chosen as a control condition as a result of clinical evidence. It is doubtful whether patients with ideomotor apraxia could perform a complex task that could be a good control in terms of neuroimaging experiments. Indeed, although controversial, some researchers emphasise that ideomotor apraxic behaviours are frequently seen in test sessions, but not in everyday life. ${ }^{46}$ This observation indicates that patients with ideomotor apraxia have difficulty in motor responses on request, but not in a self initiated movement. Thus, we thought that the control task for ideomotor praxis should be a rather simple, easy, and automatic movement that is not triggered by external stimuli. It is on such grounds that we would claim that the finger bending/unbending movement task is an appropriate control motor task to test against the ideomotor praxis task. Because we optimised the experimental design to detect the commonality of two praxic movements, the difference in activation by the two praxic movements is less informative; they are mixtures of the effect of visual/auditory information processes and imitation/verbal command specific cognitive processes.
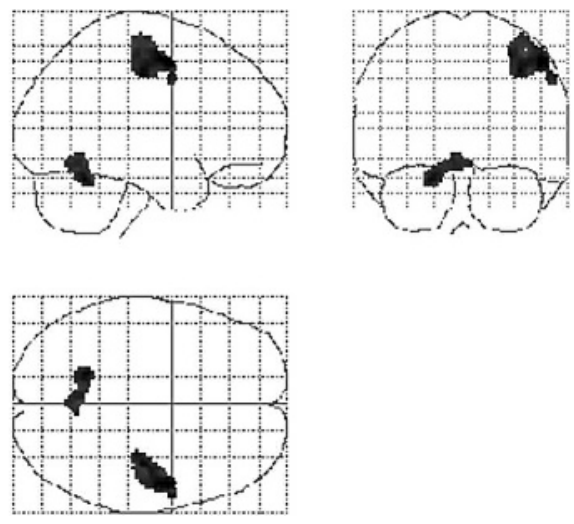
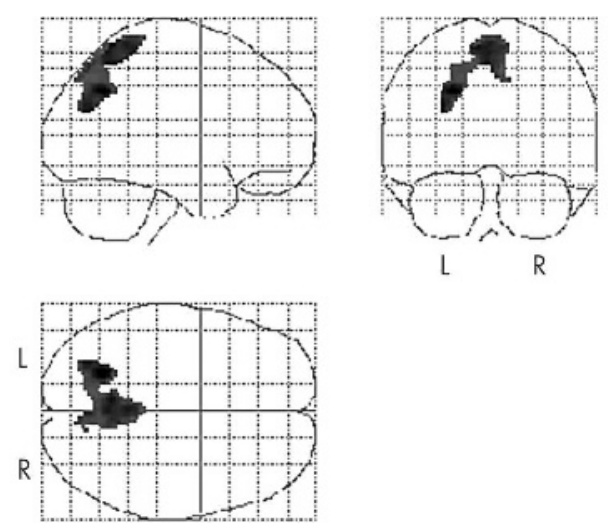

Figure 3 Statistical parametric maps of $z(S P M\{Z\} s)$ that show the neural correlates of the ideomotor praxis (left) and finger bending/unbending movements (right). SPM \{\}$_{s}$ are thresholded at $\mathrm{p}<0.05$ (corrected for multiple comparisons). Left: activation revealed by the detection of signal increase in $(I+V C) / 2$ against FB/UM, masked by l against REST, VC against REST, I against FB/UM, VC against FB/UM. Right: activation revealed by the detection of signal increase in FB/UM against $(\mathrm{I}+\mathrm{VC}) / 2$, masked by FB/UM against REST, FB/UM against I, FB/UM against VC. The SPM $\{Z\} \mathrm{S}$ are displayed on a glass brain viewed from three directions. The transverse projection is viewed from the top, and the coronal projection is viewed from the back (the right of the map corresponds to the right of the brain). Tables 5 and 6 summarise the local maxima of the SPM $\{Z\}$ s. FB/UM, finger bending/ unbending movement; I, imitation; VC, verbal command. 
Table 4 The local maxima of activated regions in VC against I, masked by VC against REST

\begin{tabular}{|c|c|c|c|c|c|}
\hline \multirow[b]{2}{*}{ Activated region } & \multirow[b]{2}{*}{$L / R$} & \multicolumn{3}{|c|}{ Coordinates } & \multirow[b]{2}{*}{$Z$ value } \\
\hline & & $\mathbf{x}$ & y & z & \\
\hline Superior temporal gyrus & L & $\begin{array}{l}-66 \\
-50 \\
-60\end{array}$ & $\begin{array}{l}-22 \\
-28 \\
-6\end{array}$ & $\begin{array}{l}2 \\
4 \\
-8\end{array}$ & $\begin{array}{l}6.75 \\
5.54 \\
4.57\end{array}$ \\
\hline Superior temporal gyrus & $\mathrm{R}$ & $\begin{array}{l}66 \\
62 \\
60\end{array}$ & $\begin{array}{l}-16 \\
-4 \\
12\end{array}$ & $\begin{array}{l}-2 \\
-6 \\
-10\end{array}$ & $\begin{array}{l}6.37 \\
5.36 \\
5.12\end{array}$ \\
\hline
\end{tabular}

I, imitation; L, left; R, right; VC, verbal command.

\section{RESULTS}

Analysis 1. I against FB/UM, masked by I against REST Analysis 1 revealed activation in the posterior part of the left intraparietal sulcus and the bilateral precunei (table 1; fig 2A). Because the imitation condition involved visual processing, activation in the dorsal and ventral occipital areas was prominent: the fusiform gyrus, lingual gyrus, inferior occipital gyrus, middle occipital gyrus, inferior temporal gyrus, lateral geniculate nucleus, and pulvinar were activated bilaterally.

\section{Analysis 2. I against VC, masked by I against REST}

In analysis 2, we found activation in the dorsal and ventral occipital areas noted above, but not in the parietal areas (table 2; fig 2B).

\section{Analysis 3. VC against FB/UM, masked by VC against REST}

In this analysis, activity was detected in the posterior part of the left intraparietal sulcus, bilateral precunei, and bilateral superior temporal gyri (table 3; fig 2C). The superior temporal regions in both hemispheres included Heschel's gyrus and its surrounding region, but the left region extended more posteriorly than the right, and the right region extended more anteriorly than the left.
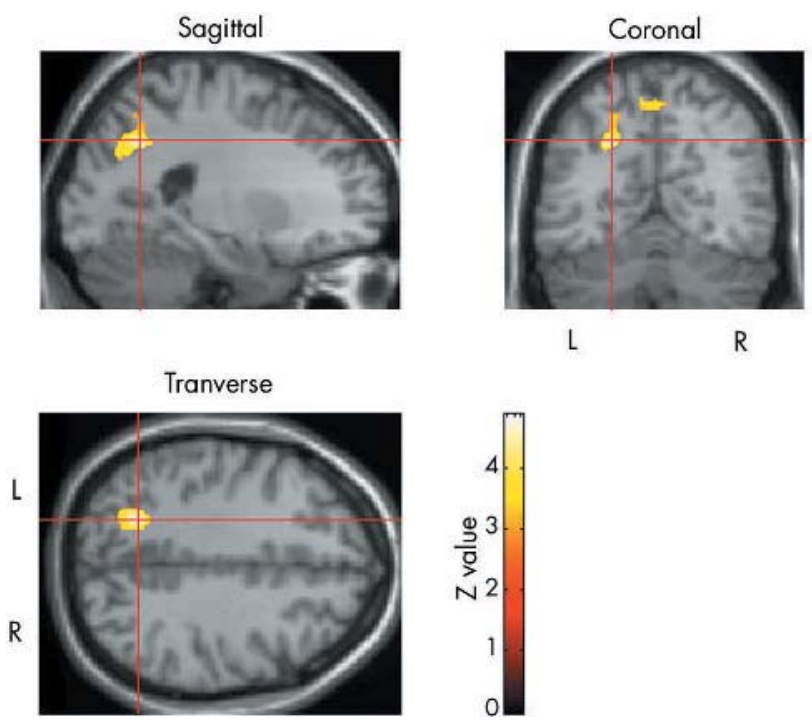

Analysis 4. VC against I, masked by VC against REST Only the superior temporal gyrus was activated bilaterally in this analysis (table 4; fig 2D). The superior temporal regions in both hemispheres included Heschel's gyrus and its surrounding region, but the left region extended more posteriorly than the right, and the right region extended more anteriorly than the left, as in analysis 3.

\section{Analysis $5 .(I+V C) / 2$ against $F B / U M$, masked by I against REST, VC against REST, I against FB/UM, VC against $F B / U M$}

This analysis was carried out to reveal regions that were activated in common in two ideomotor praxis relative to the finger bending/unbending movement. The depth of the posterior part of the left intraparietal sulcus and bilateral precunei were the detected regions (table 5; figs 3, 4).

Analysis 6. FB/UM against $(I+V C) / 2$, masked by FB/ UM against REST, FB/UM against I, FB/UM against VC This analysis showed activation in the right hand motor area, left cerebellar hemisphere, and vermis (table 6; fig 3).

\section{DISCUSSION}

We found that the depth of the posterior part of the left intraparietal sulcus was a common region activated by two
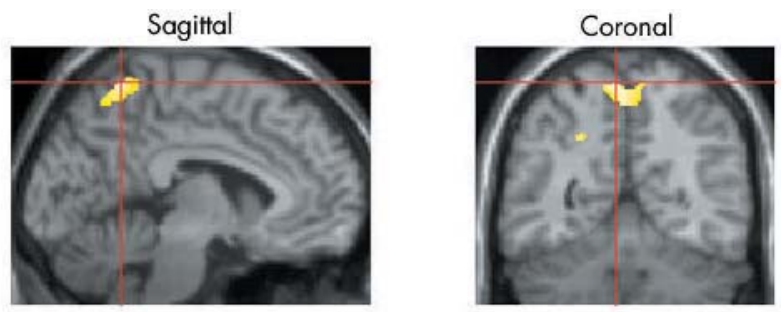

L

$\mathrm{R}$

$\mathrm{L}$

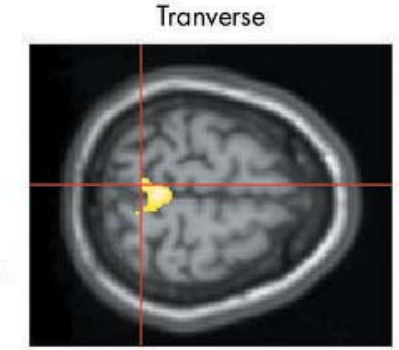

Figure 4 Regions activated in common for two kinds of ideomotor praxis relative to finger bending/unbending movements. The detection of signal increase in $((I+V C) / 2$ against $F B / U M$, masked by I against REST, VC against REST, I against FB/UM, VC against FB/UM) showed activation in the depth of the posterior part of the left intraparietal sulcus and bilateral precunei as the neural substrate of the ideomotor praxis. The statistical parametric maps of $z$ (SPM ZZ $\}$ s) are overlaid on the three direction sections of a normalised T1 template brain. SPM $\{Z\}$ s are thresholded at $p<0.05$ (corrected for multiple comparisons). The crosshair indicates the position of the local maxima of $Z$ values in the cluster. Left: activation of the depth of the posterior part of the left intraparietal sulcus. The coordinate was $(-24,-62,38)$. Right: activation of the precuneus. The coordinate was $(-6,-56,66)$. The scale for the $Z$ value is shown. Table 5 summarises the local maxima of the SPM ZZ\}s. FB/UM, finger bending/unbending movement; I, imitation; VC, verbal command. 


\begin{tabular}{|c|c|c|c|c|c|}
\hline \multirow[b]{2}{*}{ Activated region } & \multirow[b]{2}{*}{$L / R$} & \multicolumn{3}{|c|}{ Coordinates } & \multirow[b]{2}{*}{$Z$ value } \\
\hline & & $\bar{x}$ & $y$ & $z$ & \\
\hline Intraparietal sulcus & $\mathrm{L}$ & $\begin{array}{l}-24 \\
-30 \\
-18\end{array}$ & $\begin{array}{l}-62 \\
-70 \\
-68\end{array}$ & $\begin{array}{l}38 \\
28 \\
52\end{array}$ & $\begin{array}{l}4.89 \\
4.27 \\
3.56\end{array}$ \\
\hline Precuneus & $\begin{array}{l}L \\
R\end{array}$ & $\begin{array}{l}-6 \\
6 \\
2 \\
4\end{array}$ & $\begin{array}{l}-56 \\
-56 \\
-70 \\
-76\end{array}$ & $\begin{array}{l}66 \\
64 \\
52 \\
46\end{array}$ & $\begin{array}{l}4.48 \\
4.07 \\
3.95 \\
3.57\end{array}$ \\
\hline
\end{tabular}

$F B / U M$, finger bending/unbending movement; I, imitation; L, leff; R, right; VC, verbal command.

ideomotor praxic movements; that is, imitation and movements executed by verbal command. This region can be considered as a common neural substrate for processing the visual and auditory command to generate the ideomotor praxic motor response. A functional neuroimaging study of a patient with apraxia using fludeoxyglucose F 18 positron emission tomography (PET) reported focal reductions of glucose metabolism in the left angular gyrus and left supramarginal gyrus. ${ }^{27}$ Heilman and colleagues proposed a model of ideomotor apraxia in which the movement formulae for ideomotor praxis are stored in the left inferior parietal lobule based on lesion data. ${ }^{13}$ Our finding fits well with a recent lesion study using computed tomography (CT) and MRI by Haaland and colleagues, who found maximal overlap of lesions in patients with ideomotor apraxia in the left intraparietal sulcus. ${ }^{8}$ More focused localisation in our study and that of Haaland et al might be the result of the better resolution in the more recent CT and MRI studies, compared with PET or old lesion studies. Our finding does not necessarily disagree with earlier studies reporting white matter lesions in ideomotor apraxia, because the posterior part of the left intraparietal sulcus is thought to be a part of the brain network related to ideomotor praxis.

We think that the attentive component is inevitably involved in ideomotor praxis. This attentional component is crucial for ideomotor praxis, because a deficit in prompt action in response to commands, often out of context, is one of the important aspects of ideomotor praxis. Attention in motor responses has been studied by neuroimaging, and these studies revealed frontoparietal activation..$^{28}{ }^{29}$ However, we found activation for ideomotor praxic movements only in the left parietal region rather than the frontoparietal network, ${ }^{30}$ and this implies that the depth of the posterior part of the left intraparietal sulcus has a specific role in ideomotor praxic movements rather than in more general motor attention.

Interestingly, an fMRI study showed that this region is also involved in the "tool use pantomime", which is traditionally carried out in the test for ideational apraxia. ${ }^{31}$ That study measured BOLD signal increases in the tool use pantomime by giving names of tools auditorily against repetition of sequenced hand movements. This coincidence suggests that the region corresponds to the notion of "praxis", which may be regarded as a cognitive activity in which external stimuli are mapped into appropriate motor responses.

We found the right primary motor area, left cerebellar hemisphere, and vermis to be specific neural correlates of finger bending/unbending movements. The increased activity of these areas relative to that in ideomotor praxis may reflect the more frequent movements in the FB/UM condition, compared with the I or VC conditions, where the movements were generated every four seconds.

We found activation in the precuneus bilaterally in analysis that extracts the neuroanatomical correlates of the two ideomotor praxis. It is not clear whether lesions to the bilateral precunei cause ideomotor apraxia, but this result may explain ideomotor apraxia after right brain damage in right handers. ${ }^{32}$ Because several neuroimaging studies have shown participation of the precuneus in complex finger movements $^{33}$ and in motor imagery generation, ${ }^{34} 35$ the bilateral precunei may be involved in ideomotor praxis. Elucidating the exact role of the precuneus in ideomotor praxis is an issue for future studies.

Recent neuroimaging studies have claimed that the neural substrate for imitation is the left Brodmann area 44, which is a part of Broca's area. ${ }^{11}{ }^{12}$ Krams and colleagues reported activation of Broca's area in a key press imitation task and they attributed the activation of Broca's area to copying movement. ${ }^{11}$ In their study, a sketch of a right hand presented on a screen indicated which finger to move by darkening of the nail colour and the subject pressed the keys sequentially, following the changes in nail colour. This task is not appropriate as an imitation task, because the hand sketch was kept still throughout the experiment. In Iacoboni and colleagues' experiment, ${ }^{12}$ an animated hand was displayed and the index or the middle finger of the animated hand was lifted at random. The subject was instructed to imitate the movement with the right hand (imitation task). In a control

Table 6 The local maxima of activated regions in $\mathrm{FB} / \mathrm{UM}$ against $(\mathrm{I}+\mathrm{VC}) / 2$, masked by $F B / U M$ against $R E S T, F B / U M$ against $I, F B / U M$ against $V C$

\begin{tabular}{|c|c|c|c|c|c|}
\hline \multirow[b]{2}{*}{ Activated region } & \multirow[b]{2}{*}{ Left/Right } & \multicolumn{3}{|c|}{ Coordinates } & \multirow[b]{2}{*}{$Z$ value } \\
\hline & & $x$ & $y$ & $z$ & \\
\hline Precentral gyrus & $R$ & $\begin{array}{l}50 \\
38 \\
32\end{array}$ & $\begin{array}{l}-2 \\
-18 \\
-22\end{array}$ & $\begin{array}{l}50 \\
46 \\
56\end{array}$ & $\begin{array}{l}4.49 \\
4.14 \\
3.83\end{array}$ \\
\hline $\begin{array}{l}\text { Cerebellar hemisphere } \\
\text { Vermis }\end{array}$ & L & $\begin{array}{l}-18 \\
-4 \\
6\end{array}$ & $\begin{array}{l}-54 \\
-58 \\
-60\end{array}$ & $\begin{array}{l}-22 \\
-12 \\
-12\end{array}$ & $\begin{array}{l}4.14 \\
3.78 \\
3.45\end{array}$ \\
\hline
\end{tabular}


task, a static hand was displayed and a cross appeared on the index or the middle finger at random (symbolic instruction task). The subject was instructed to lift the corresponding finger of the right hand in response to the cross. Broca's area was activated not only in the imitation task, but also in a finger movement task with symbolic instruction. The two neuroimaging studies used sequential finger movements as imitation tasks, which involve observation and retention of information about the movements in sequence. Therefore, the function of Broca's area may be considered in both studies as a temporary storage of information for sequential movement, but not for imitation, as noted in our previous work. ${ }^{36}$

In the statistical analysis that extracts regions subserving imitation (I against FB/UM, masked by I against REST), the posterior part of the left intraparietal sulcus, the bilateral precunei, fusiform gyri, lingual gyri, inferior occipital gyri, middle occipital gyri, inferior temporal gyri, lateral geniculate nuclei, and pulvinar were revealed, but when we analysed the signal increase in I against VC, only the dorsal and ventral occipital areas were detected. These results mean that the left parietal region was used not only for imitation, but also for the movements executed by verbal command. The analysis for regions activated in common in I and VC supported this interpretation.

A few other neuroimaging studies have investigated brain mechanisms of imitation. ${ }^{37-39}$ They all reported involvement of the left inferior parietal lobe in producing imitation. They matched the input stimuli but not the output movements in their control conditions; their control conditions were watching a fixation point ${ }^{37}$ and free response at will. ${ }^{38}{ }^{39}$ In contrast, the I and VC conditions have identical movement in our study. Because we did not detect activation in the left inferior parietal lobe by the detection of signal increase in the I condition against the VC condition, this region may not have a specific role in imitation.

Imitation involves cognitive components that movements executed by verbal command do not have-for example, analysing the posture and generating the motor pattern corresponding to the presented posture. Some of these components seem to be processed in the dorsal and ventral occipital area. Hermsdörfer and colleagues compared brain activation during discrimination of hand or finger gestures with that seen during discrimination of persons. ${ }^{40}$ Activation of the parietal cortices and the occipital cortices was detected even when the visual stimuli were identical. Based on their results and ours, we reason that imitation specific cortices, especially for visual analysis of hand postures, may be found in the common part of the occipital region detected in our study and that of Hermsdörfer et al; namely, a part of the inferior temporal/occipital gyri.

When we detected signal increases in VC against FB/UM, the depth of the posterior part of the left intraparietal sulcus and bilateral precunei were activated. From the observation that these regions were also found in the corresponding analyses for movement by imitation, we think that the functions of these regions are not specific for movements executed by verbal command. The bilateral superior temporal gyri were activated selectively by movements executed by verbal command. However, with our experimental design we cannot determine whether some of the cognitive components involved in movements executed by verbal command, such as recall of the movement, are processed in the bilateral superior temporal gyri, because mere word listening tasks activate the same regions. ${ }^{41}$

\section{ACKNOWLEDGEMENTS}

This work was supported by Grant L-2-12 from the Japan Society for the Promotion of Science.

\section{Authors' affiliations}

M Makuuchi, M Sugishita, Cognitive Neuroscience, Faculty of Medicine, University of Tokyo, 7-3-1 Hongo, Bunkyo-ku, Tokyo 113-0033, Japan T Kaminaga, Radiology, Teikyo University Hospital, 2-11-1 Kaga, Itabashi-ku, Tokyo 173-8606, Japan

M Sugishita, Institute of Brain and Blood Vessels, 366 Ohtamachi, Isesaki, Gunma 372-0006, Japan

Competing interests: none declared

\section{REFERENCES}

1 Liepmann H. Das Krankheitsbild der Apraxie (Motorische Asymbolie) auf Grund eines Falles von einseitiger Apraxie). Monatsschrift für Psychiatrie und Neurologie 1990;8:15-44, 102-132, 182-197.

2 Geschwind N, Damasio AR. Apraxia. In: Frederiks JAM, ed. Handbook of clinical neurology, Vol.1. Amsterdam: Elsevier Science Publishers, 1985:423-32.

3 De Ajuriaguerra J, Hecaen $\mathrm{H}$, Angelergues R. Les apraxies variétés cliniques et latéralisation lésionelle. Rev Neurol 1960;102:566-94.

4 De Renzi E, Faglioni P, Sorgato P. Modality-specific and supramodal mechanisms of apraxia. Brain 1982;105:301-12.

5 De Renzi E. Apraxia. In: Boller F, Grafman J, eds. Handbook of neuropsychology, Vol.2. Amsterdam: Elsevier, 1989:245-63.

6 Ochipa C, Rothi U, Heilman KM. Conduction apraxia. J Neurol Neurosurg Psychiatry 1994;57:1241-4.

7 Ghika J, Ghika-Schmid F, Bogousslasvky J. Parietal motor syndrome: a clinical description in 32 patients in the acute phase of pure parietal strokes studied prospectively. Clin Neurol Neurosurg 1998;100:271-82.

8 Haaland KY, Deborah L, Harrington DL, et al. Neural representations of skilled movement. Brain 2000;123:2306-13.

9 Kertesz A, Ferro JM. Lesion size and location in ideomotor apraxia. Brain 1984; 107:921-33.

10 Pramstaller PP, Marsden CD. The basal ganglia and apraxia. Brain 1996; 119:319-40.

11 Krams M, Rushworth MF, Deiber MP, et al. The preparation, execution and suppression of copied movements in the human brain. Exp Brain Res 1998; 120:386-98.

12 lacoboni M, Woods RP, Brass $M$, et al. Cortical mechanisms of human imitation. Science 1999;286:2526-8.

13 Heilman KM, Watson RT, Rothi LG. Disorders of skilled movements: limb apraxia. In: Feinberg TE, Farah MJ, eds. Behavioral neurology and neuropsychology. New York: McGraw-Hill, 1997:227-35.

14 Oldfield RC. The assessment and analysis of handedness: the Edinburgh inventory. Neuropsychologia 1971;9:97-113.

15 Sperry RW. Hemisphere disconnection and unity in conscious awareness. Am Psychol 1968;23:723-33.

16 Liepmann H. Apraxie. Ergebnisse der Gesmten Medizin 1920;1:516-43.

17 Friston KJ, Jezzard P, Turner R. Analysis of fMRI time-series. Hum Brain Mapp 1994;1:153-71.

18 Friston KJ, Holmes AP, Poline JP, et al. Analysis of FMRI time-series revisited. Neuroimage 1995;2:45-53.

19 Friston KJ, Holmes AP, Worsley KJ, et al. Statistical parametric maps in functional imaging: a general linear approach. Hum Brain Mapp 1995;2:189-210.

20 Worsley KJ, Friston KJ. Analysis of FMRI time-series revisited-again. Neuroimage 1995;2:173-81.

21 Mazziotta JC, Toga AW, Evans A, et al. A probabilistic atlas of the human brain: theory and rational for its development. Neuroimage 1995;2:89-101.

22 Talairach J, Tournoux P. Co-planar stereotaxic atlas of the human brain. Stuttgart: George Thieme Verlag, 1988.

23 Holmes AP, Friston KJ. Generalisability, random effects and population inference. Neuroimage 1998;7:S754.

24 Perani D, Fazio F, Borghese NA, et al. Different brain correlates for watching real and virtual hand actions. Neuroimage $2001 ; 14: 749-58$.

25 Devlin JT, Russell RP, Price CJ, et al. Is there an anatomical basis for categoryspecificity? Semantic memory studies in PET and fMRI. Neuropsychologia 2002;40:54-75.

26 De Renzi E, Motto F, Nichelli P. Imitating gestures. A quantitative approach to ideomotor apraxia. Arch Neurol 1980;37:6-10.

27 Kareken DA, Unverzagt F, Caldemeyer K, et al. Functional brain imaging in apraxia. Arch Neurol 1998;55:107-13.

28 Jueptner M, Stephan KM, Frith CD, et al. Anatomy of motor learning I. Frontal cortex and attention to action. J Neurophysiol 1997;77:1313-24.

29 Rowe J, Stephan KE, Friston K, et al. Attention to action in Parkinson's disease: impaired effective connectivity among frontal cortical regions. Brain 2002; 125:276-89.

30 Corbetta M, Shulman GL. Control of goal-directed and stimulus-driven attention in the brain. Nat Rev Neurosci 2002;3:201-15.

31 Moll J, De Oliveira-Souza R, Passman L, et al. Functional MRI correlates of real and imagined tool-use pantomimes. Neurology 2000;54:1331-6.

32 Heilman KM, Rothi LG, Mack L, et al. Apraxia after a superior parietal lesion. Cortex 1986;22:141-50.

33 Catalan MJ, Honda M, Weeks RA, et al. The functional neuroanatomy of simple and complex sequential finger movements: a PET study. Brain 1998; 121:253-64. 
34 Ogiso T, Kobayashi K, Sugishita M. The precuneus in motor imagery: magnetoencephalographic study. Neuroreport 2000;1 1:1345-9.

35 Stephan KM, Fink GR, Passingham RE, et al. Functional anatomy of the mental representation of upper extremity movements in healthy subjects. J Neurophysiol 1995:73:373-86.

36 Makuuchi M, Kaminaga T, Sugishita M. Both parietal lobes are involved in drawing: a functional MRI study and implications for constructional apraxia. Brain Res Cogn Brain Res 2003; 16:338-47.

37 Tanaka S, Invi T, Iwaki S, et al. Neural substrates involved in imitating finger configurations: an fMRI study. Neuroreport 2001;12:1171-4.
38 Chaminade T, Meltzoff AN, Decety J. Does the end justify the means? A PET exploration of the mechanisms involved in human imitation. Neuroimage 2002; 15:318-28.

39 Decety J, Chaminade T, Grezes J, et al. A PET exploration of the neural mechanisms involved in reciprocal imitation. Neuroimage 2002;15:265-72.

40 Hermsdörfer J, Goldenberg G, Wachsmuth C, et al. Cortical correlates of gesture processing: clues to the cerebral mechanisms underlying apraxia during the imitation of meaningless gestures. Neuroimage 2001;14:149-61.

41 Wise RJS, Scott SK, Blank SC, et al. Separate neural subsystems within "Wernicke's area". Brain 2001;124:83-95.

\section{Clinical Evidence-Call for contributors}

Clinical Evidence is a regularly updated evidence-based journal available worldwide both as a paper version and on the internet. Clinical Evidence needs to recruit a number of new contributors. Contributors are healthcare professionals or epidemiologists with experience in evidence-based medicine and the ability to write in a concise and structured way.

Areas for which we are currently seeking authors:

- Child health: nocturnal enuresis

- Eye disorders: bacterial conjunctivitis

- Male health: prostate cancer (metastatic)

- Women's health: pre-menstrual syndrome; pyelonephritis in non-pregnant women

However, we are always looking for others, so do not let this list discourage you.

Being a contributor involves:

- Selecting from a validated, screened search (performed by in-house Information Specialists) epidemiologically sound studies for inclusion.

- Documenting your decisions about which studies to include on an inclusion and exclusion form, which we keep on file.

- Writing the text to a highly structured template (about 1500-3000 words), using evidence from the final studies chosen, within 8-10 weeks of receiving the literature search.

- Working with Clinical Evidence editors to ensure that the final text meets epidemiological and style standards.

- Updating the text every six months using any new, sound evidence that becomes available. The Clinical Evidence in-house team will conduct the searches for contributors; your task is simply to filter out high quality studies and incorporate them in the existing text.

- To expand the topic to include a new question about once every 12-18 months.

If you would like to become a contributor for Clinical Evidence or require more information about what this involves please send your contact details and a copy of your CV, clearly stating the clinical area you are interested in, to Klara Brunnhuber (kbrunnhuber@ bmigroup.com).

\section{Call for peer reviewers}

Clinical Evidence also needs to recruit a number of new peer reviewers specifically with an interest in the clinical areas stated above, and also others related to general practice. Peer reviewers are healthcare professionals or epidemiologists with experience in evidence-based medicine. As a peer reviewer you would be asked for your views on the clinical relevance, validity, and accessibility of specific topics within the journal, and their usefulness to the intended audience (international generalists and healthcare professionals, possibly with limited statistical knowledge). Topics are usually 1500-3000 words in length and we would ask you to review between 2-5 topics per year. The peer review process takes place throughout the year, and our turnaround time for each review is ideally 10-14 days.

If you are interested in becoming a peer reviewer for Clinical Evidence, please complete the peer review questionnaire at www.clinicalevidence.com or contact Klara Brunnhuber (kbrunnhuber@bmigroup.com). 